\title{
Risk factors for catheter-related bloodstream infection: a prospective multicenter study in Brazilian intensive care units
}

Authors

Daniela Bicudo ${ }^{1}$

Ruth Batista ${ }^{2}$

Guilherme Henrique

Furtado $^{3}$

Angela Sola

Eduardo Alexandrino Servolo de Medeiros ${ }^{4}$

${ }^{1}$ MSc; Nurse, Hospital São Paulo, Universidade Federal de São Paulo (UNIFESP), SP, Brazil

${ }^{2} \mathrm{PhD}$; Nurse, Hospital São Paulo, UNIFESP, SP, Brazil ${ }^{3}$ Post-doctorate in Health Sciences, Infectology, Hospital São Paulo, UNIFESP, SP, Brazi ${ }^{4}$ Professor, $\mathrm{PhD}$, Infectious Diseases Department, UNIFESP, SP, Brazil
Submitted on: 01/05/2011 Approved on: 02/17/2011

Correspondence to: Eduardo Alexandrino Servolo de Medeiros Rua Napoleão de Barros, 690 - $2^{\circ}$ andar 04024-002, Vila Clementino São Paulo, SP Brazil edubala@netpoint.com.br

We declare no conflict of interest.

(C2011 Elsevier Editora Ltda All rights reserved.

\begin{abstract}
Introduction: Central venous catheters (CVC) are devices of great importance in health care. The advantages gained from the use of catheters outweigh the complications that might result from their use, among which bloodstream infections (BSI). In spite of its importance, few national studies have addressed this issue. Objective: The aim this study was to determine the incidence of BSI in patients with CVC, hospitalized in ICU, as well as the variables associated with this complication. Methods: Multicentric cohort study carried out at ICUs of three hospitals at Universidade Federal de São Paulo complex. Results: A total of 118 cases of BSI in 11.546 catheters day were observed: $10.22 \mathrm{BSI}$ per 1,000 catheters day. On average, BSI was associated to seven additional days of hospital stay in our study $(\mathrm{p}<0.001)$, with a significant difference between types of catheters. Concerning the place of insertion, there was no statistical difference in BSI rates. Conclusion: We concluded that a patient who uses a catheter for longer than 13 days presents a progressive risk for infection of approximately three times higher in relation to a patient who uses the catheter for less than 13 days $(\mathrm{p}<0.001)$. The median duration of catheter use was 14 days among patients with BSI and 9 days in patients without infection $(\mathrm{p}<0.001)$. There was higher prevalence of Gram-negative infections. The risk factors for BSI were utilization of multiple-lumen catheters, duration of catheterization and ICU length of stay.
\end{abstract}

Keywords: intensive care units; catheter-related infections; catheterization, BSI.

\section{INTRODUCTION}

Intravascular catheters are devices of great importance for healthcare and are essential for adequate management of critically ill patients admitted to intensive care units (ICU). ${ }^{1}$

The advantages of using catheters are outweight their complications, which are mainly bloodstream infections (BSI). In ICUs, central lines may be of long term use, which exponentially increases patients' colonization potential by resistant microorganisms secondary to increasing catheter manipulation. Such associated factors can increase the risk of acquiring BSIs. ${ }^{2}$

BSI are among the most frequent nosocomial infections and it is associated with significant morbidity and mortality. Consequently, the costs of treatment are increased from $\$ 6,000$ to over $\$ 90,000$ for those with BSI. ${ }^{3}$

The incidence of BSI varies considerably according to many factors. It may also be influenced by the patients' characteristics, including underlying diseases and age, and by parameters related to the catheters, such as the conditions under which they were inserted (e.g. urgent versus non-urgent), the type of the catheter (tunneled versus non-tunneled), and the insertion location (subclavian versus jugular vein). ${ }^{2}$

The objective of this study was to determine the potential risk factors for BSI in patients with a central venous catheter admitted to six ICUs from three hospitals in São Paulo, Brazil.

\section{MATERIAL AND METHODS}

\section{Setting}

A multicenter study was undertaken during a 12-month period (November 2005 to October 2006) at six medical-surgical ICUs from three hospitals in São Paulo, Brazil. Each hospital had an infection control team comprised of an infectious disease physician and an infection control nurse, who were responsible for nosocomial infection surveillance. 


\section{Inclusion criteria}

All patients over 18 years old admitted to the particpating ICUs during the study period who had a non-tunelled central venous catheter (CVC) inserted were eligible for the study. In addition, these patients were observed either for 48 hours after discharge from the ICU or after the withdrawal of catheter if they remained in the ICU. The number of triple-lumen central venous catheters used in this study was small, thus, we grouped them as multiple-lumen catheters.

\section{Data collection}

The epidemiological surveillance was performed by the infection control nurses team at the participating institutions. The nurses received a 45-minute theoretical and practical training for with the objective of standardizing the completion of the epidemiological surveillance form used in this study. The form was completed for each patient with CVC in the study ICUs and included information on risk factors and CVC characteristics. BSI incidence was assessed using the National Nosocomial Infections Surveillance (NNIS) definition and was sent monthly to the person responsible for the study.

\section{Definitions}

Catheter-related BSI (CR-BSI) surveillance was conducted for all patients in the ICUs using definitions from the Centers of Diseases Control and Prevention (CDC). ${ }^{2}$

\section{Microbiological procedures}

Collection of blood cultures was performed in agreement with the standardized method used by the infection control team from each institution. Briefly, this included hand washing before the procedure, antisepsis of the puncture local area with $10 \%$ povidone or chlorhexidine gluconate, and the use of gloves. Both the processing and detection of microorganisms' growth in the blood cultures were performed using an automated technique with BACTEC ${ }^{\circledast} 9240$ system (Becton Dickson Diagnostic Instrument System, EUA).

\section{Statistical analysis}

For univariate analysis, the chi-square test was used or the Fischer's exact test when indicated. Continuous variables were analyzed by the Mann-Whitney/Wilcoxon test or Student's $t$ test if the distribution was normal. In all tests, the significance level was fixed at $5 \%$.

Multivariate analysis was performed through a logistic regression model with a significance level of 5\%. The program "Statistical Package for the Social Science" for Windows version 10.0 (SPSS) was used.

\section{RESULTS}

During the study period between November 01, 2005 and October 31, 2006, 928 CVCs inserted in 555 patients were assessed.
Three hundred thirty-two patients (59.8\%) were male and 223 patients $(40.7 \%)$ were female; the mean age was 53.9 years; the average length of ICU stay was 24.4 days; the mean APACHE II score was 16 (range, 10-34). Three hundred ninety-three $(70.8 \%)$ patients had a clinical diagnosis and $162(29.1 \%)$ patients had a surgical diagnosis.

The CVC utilization rate was 1.67 per patient. Regarding central venous catheter type, 621 (66.9\%) were multiple-lumen catheters, and 307 (33.1\%) were single-lumen catheters. As to the insertion location, 609 (65.6\%) CVC were inserted in the subclavian vein, $306(32.9 \%)$ in the jugular vein, and $13(1.4 \%)$ in the femoral vein. Seven hundred thirty-two $(78.8 \%)$ catheters were inserted in the ICU, $151(16.2 \%)$ in the surgical room and $45(4.8 \%)$ in other hospital units. The average duration of catheterization in each patient was 12.4 days (standard deviation \pm 18.4 ).

A total of 11,546 catheters/day and 118 BSI were identified. One hundred six $(89.8 \%)$ were laboratoryconfirmed and $12(10.2 \%)$ were clinical sepsis. The BSI incidence rate was 10.2 per 1,000 catheters/day.

The most prevalent microorganisms were Acinetobacter baumannii ( $\mathrm{n}=20 ; 19 \%)$, Pseudomonas aeruginosa $(\mathrm{n}=16 ; 15.2 \%)$, Klebsiella pneumoniae $(\mathrm{n}=16 ; 15.24 \%)$, coagulase-negative staphylococci $(\mathrm{n}=14 ; 13.3 \%)$, Enterococcus faecium ( $\mathrm{n}=9 ; 8.5 \%)$ and Staphylococcus aureus $(\mathrm{n}=7 ; 6.6 \%)$.

The sole patient-related risk factor for bloodstream infection was the length of ICU stay. Age, gender, underlying diagnosis and APACHE II score were not significantly associated with BSI. Table 1 shows the results of the univariate analysis.

Concerning the variables related to CVC, we observed a significant difference of BSI rates between single-lumen catheters compared to multiple-lumen catheters. There was a significant difference with regard to the duration of CVC use in univariate analysis as well. Table 2 displays these results.

Table 1. Univariate analysis of patient characteristics

\begin{tabular}{lccc}
\hline Characteristics & $\begin{array}{c}\text { BSI } \\
(\mathbf{n}=\mathbf{1 0 7})\end{array}$ & $\begin{array}{c}\text { Without BSI } \\
(\mathbf{n}=448)\end{array}$ & $\mathbf{p}$ \\
\hline Age $>$ 60 years & $54(50.4)$ & $188(41.9)$ & 0.13 \\
\hline Male gender & $63(58.8)$ & $269(60.0)$ & 0.91 \\
\hline Surgery & $26(24.3)$ & $136(30.3)$ & 0.26 \\
\hline APACHE II score, mean & 15.7 & 15.4 & 0.39 \\
\hline $\begin{array}{l}\text { Length of ICU stay, } \\
\text { mean, days }\end{array}$ & 24 & 17 & $<0.001$ \\
\end{tabular}

BSI, bloodstream infection; ICU, intensive care unit. 
Table 2. Univariate analysis of central venous catheter characteristics

\begin{tabular}{lccc}
\hline Characteristics & \multicolumn{1}{c}{$\begin{array}{c}\text { BSI } \\
(\mathbf{n = 1 1 8})\end{array}$} & $\begin{array}{c}\text { Without BSI } \\
(\mathbf{n}=\mathbf{8 1 0})\end{array}$ & $\mathbf{p}$ \\
\hline $\begin{array}{l}\text { Multiple-lumen } \\
\text { catheter use }\end{array}$ & $92(77.9)$ & $529(65.3)$ & 0.009 \\
\hline $\begin{array}{l}\text { Catheter location } \\
\text { in subclavian vein }\end{array}$ & $79(66.9)$ & $530(65.4)$ & 0.46 \\
\hline $\begin{array}{l}\text { Duration of } \\
\text { catheterization, } \\
\text { median, days }\end{array}$ & 14 & 9 & $<0.001$ \\
$\begin{array}{l}\text { More than one } \\
\text { CVC utilized }\end{array}$ & $52(44 \%)$ & $321(39.6 \%)$ & 0.41 \\
\hline
\end{tabular}

BSI, bloodstream infection; CVC, central venous catheter.

In multivariate analysis only use of multiple-lumen catheters (OR 2.86 95\% CI 1.69-4.85, p < 0.001) and duration of CVC catheterization (OR 2.35 95\% CI 1.26-4.39, p = 0.007) were found to be independent risk factors for CR-BSI.

\section{DISCUSSION}

In our study, the bloodstream infection rate $(10.2$ per 1,000 catheters/day) was greater than the average rate previously reported from U.S. ICUs. ${ }^{2,4}$

In 2007, we started to report infection rates from our hospitals to the International Nosocomial Infection Control Consortium (INNIC). This study involved 98 ICUs from developing countries (Latin America, Asia, Africa, and Europe). These countries have similar difficulties, for example: hand hygiene compliance is very low, receives limited financial or administrative support, and the nurse-to-patient staffing ratios in hospitals are low. This study reported a bloodstream infection rate (9,2 per 1,000 catheters/ day) similar to the rate found in our study. ${ }^{5-7}$

Potential risk factors for BSI have been described in previous studies, e.g., type of catheter, duration of catheterization, and number of catheter lumens. Nonetheless, no study has sought to identify the potential risk factors for BSI through a multi-center study in our country.

We observed an average of seven additional days of hospital care for patients with bloodstream infection in our study. Additional days of hospital stay have been associated with higher BSI rates. ${ }^{8,9}$

In the multivariate analysis, we observed that the likelihood of acquiring a bloodstream infection was approximately two and half-fold greater when a patient had used a multiple-lumen CVC compared to a single-lumen catheter. Several studies demonstrated an increase in BSI rates in patients who used multiple-lumen catheters. ${ }^{3,10}$
It has been demonstrated that the location of insertion may be an important risk factor for the development of BSI. ${ }^{11}$ Catheters inserted in the jugular vein are more prone to colonization than those inserted in the subclavian vein. This may be related to factors that favor colonization of the skin next to jugular vein, e.g., oropharyngeal secretions, increased temperature, difficulties in catheter immobilization and dressing. ${ }^{8}$ In our study, there was no significant difference in BSI rates between insertion in the subclavian vein, the jugular vein, or the femoral vein.

We also concluded that a patient who had used a catheter for longer than 13 days presented progressive risk for infection of approximately three times higher in relation to a patient who had used the catheter for less than 13 days $(\mathrm{p}<0.001)$.

According to some studies, the duration of CVC use is the main determining factor for the development of bloodstream infections. ${ }^{2,12}$ Kooi et al., ${ }^{12}$ conducted a prospective multicenter study that demonstrated that the duration of CVC use between 5-9 days and over 10 days of catheterization increased the likelihood of acquiring bloodstream infections by 4 - and 8 -fold, respectively.

Concerning microbiological analysis, we observed a higher prevalence of Gram-negative rods. Our data was not consistent with the results of previous studies that have found a predominance of Gram-positive cocci. ${ }^{9}{ }^{12}$ The prevalence of Gram-negative rods may be related to the colonization of the hands of healthcare personnel or the lack of a closed-infusion system in our hospitals during the study period. Other previous study performed in our setting also showed a predominance of Gram-negative microorganisms causing BSI in a Pediatric ICU. ${ }^{13}$

Our study has several limitations. First, as a multi-center study, reliable surveillance data may be difficult to enforce. Second, we did not evaluate the catheter-site care after the insertion (e.g. dressing changes). Third, as an observational study, we could only demonstrate a possible association not causation. Finally, the data was in three hospitals and may not be generalizable to other settings. However, to our knowledge, this is the first multicenter study aimed at identifying risk factors for BSI in our country.

In conclusion, our study demonstrated that the risk factors for BSI were utilization of multiple-lumen catheters and duration of catheterization.

\section{ACKNOWLEDGEMENTS}

I wish to express our deep sense of gratitude to our friends Dra. Carla Guerra, Discipline of Infectology, Universidade Federal de São Paulo and Dr. Antonio Eduardo Benedito Silva, Discipline of Gastroenterology, Universidade Federal de São Paulo, for their useful guidance and suggestions, which helped us in completing this work. 


\section{REFERENCES}

1. Warren DK, Zack JE, Mayfield JL et al. The effect of an education program on the incidence of central venous catheter-associated bloodstream infection in a medical ICU. Chest 2004; 126:1612-8.

2. O'Grady NP, Alexander M, Dellinger EP et al. Healthcare Infection Control Practices Advisory Committee. Guidelines for the prevention of intravascular catheter-related infections. Centers for Disease Control and Prevention. MMWR Recomm Resp 2002; 51:1-29.

3. Moretti EW, Ofstead CL, Kristy RM et al. Impact of central venous catheter type and methods on catheter-related colonization an bacteremia. J Hosp Infection 2005; 61:139-45.

4. Trick WE, Miranda J, Evans AT et al. Prospective cohort study of central venous catheters among internal medicine ward patients. Am J Infect Control 2006; 34(10):636-41.

5. Rosenthal VD, Maki DG, Salomão R et al. International Nosocomial Infection Control Consortium. Device-associated nosocomial infections in 55 intensive care units of 8 developing countries. Ann Intern Med 2006, 145:582-91.

6. Rosenthal VD, Maki DG, Mehta A et al. The International Nosocomial Infection Control Consortium (INICC): Goals and objectives, description of surveillance methods, and operational activities. Am J Control 2008; 36(9):627-37.
7. Tacconelli E, Smith G, Hieke K et al. Epidemiology, medical outcomes and costs of catheter-related bloodstream infections in intensive care units of four European countries: literatureand registry-based estimates. J Hosp Infect 2009; 72:97-103.

8. Eggimann P, Pittet D. Infection control in the ICU. Chest 2001; 120:2059-93.

9. Mitt P, Adamson V, Loivukene K et al. Epidemiology of nosocomial bloodstream infections in Estonia. J Hosp Infect 2009; 71:365-70.

10. Templeton A, Schlegel M, Fleisch F et al. Multilumen central venous catheters increase risk for catheter-related bloodstream infection: prospective surveillance study. Infection 2008; 36:322-27.

11. Lorente L, Henry C, Martin MM, Jimenez A et al. Central venous catheter related infection in a prospective and observational study of 2,595 catheters. Crit Care 2005; 9(6):R631-5.

12. Van der Kooi TI, Boer AS, Mannien J et al. Incidence and risk factors of device-associated infections and associated mortality at the intensive care in the Dutch surveillance system. Intensive Care Med 2007; 33(2):271-8.

13. Abramczyk ML, Carvalho WB, Carvalho ES et al. Nosocomial infection in a pediatric intensive care unit in a developing country. Braz J Infect Dis 2003; 7(6):375-80. 